\title{
Sleep Deprivation Biases the Neural Mechanisms Underlying Economic Preferences
}

\author{
Vinod Venkatraman, ${ }^{1}$ Scott A. Huettel, ${ }^{1}$ Lisa Y. M. Chuah, ${ }^{2}$ John W. Payne, ${ }^{3}$ and Michael W. L. Chee ${ }^{2}$ \\ ${ }^{1}$ Department of Psychology and Neuroscience, Duke University, Durham, North Carolina 27708-0086, ${ }^{2}$ Cognitive Neuroscience Laboratory, Duke-NUS \\ Graduate Medical School, Singapore 169857, and ${ }^{3}$ Fuqua School of Business, Duke University, Durham, North Carolina 27705
}

A single night of sleep deprivation (SD) evoked a strategy shift during risky decision making such that healthy human volunteers moved from defending against losses to seeking increased gains. This change in economic preferences was correlated with the magnitude of an SD-driven increase in ventromedial prefrontal activation as well as by an SD-driven decrease in anterior insula activation during decision making. Analogous changes were observed during receipt of reward outcomes: elevated activation to gains in ventromedial prefrontal cortex and ventral striatum, but attenuated anterior insula activation following losses. Finally, the observed shift in economic preferences was not correlated with change in psychomotor vigilance. These results suggest that a night of total sleep deprivation affects the neural mechanisms underlying economic preferences independent of its effects on vigilant attention.

\section{Introduction}

Many persons living in developed societies sleep inadequately (Institute of Medicine, 2006; Centers for Disease Control and Prevention, 2009), believing that sustained wakefulness has no untoward effects. Several functional neuroimaging studies have revealed how short-term sleep deprivation (SD) can negatively affect attention (Chee et al., 2008; Tomasi et al., 2009), working memory (Chee and Choo, 2004; Habeck et al., 2004; Mu et al., 2005), and learning (Drummond et al., 2005; Sterpenich et al., 2009). Yet, it remains unclear whether and how SD shapes the very preferences that guide decision making, independently of these more general effects on cognition.

Behavioral studies suggest that SD-generated impairments in cognition lead to deficits in the overall quality of decision making (Harrison and Horne, 1999; Linde et al., 1999). More recent studies that involve making decisions under uncertainty have found that sleep-deprived persons tend toward riskier options (Harrison and Horne, 2000; Killgore et al., 2006; McKenna et al., 2007), mirroring the behavior of patients with medial frontal damage (Bechara et al., 2000). In the sole functional neuroimaging study on risky decision making with feedback, $24 \mathrm{~h}$ of SD resulted in increased nucleus accumbens activation for anticipated monetary gains, and attenuated insula activation for experienced monetary losses (Venkatraman et al., 2007).

Here, we sought to study SD-induced changes in risk preferences by using a novel, incentive-compatible, decision-

\footnotetext{
Received Aug. 22, 2010; revised Dec. 29, 2010; accepted Jan. 17, 2011.

This work was funded by grants awarded to M.W.L.C. from the Defense Science and Technology Agency Singapore (POD0713897) and the National Medical Research Council Singapore (NMRC/STaR/0004/2008). We thank Annette Chen, William Rekshan, and Michele Veldsman for their assistance in data collection and Su Mei Lee for editorial assistance.

This article is freely available online through the J Neurosci Open Choice option.

Correspondence should be addressed to Michael W. L. Chee, Duke-NUS Graduate Medical School, 8, College Road, \#06-18, Singapore 169857. E-mail: mchee@pacific.net.sg.

DOI:10.1523/JNEUROSCI.4407-10.2011

Copyright $\odot 2011$ the authors $\quad 0270-6474 / 11 / 313712-07 \$ 15.00 / 0$
}

making task (Payne, 2005; Venkatraman et al., 2009) where participants evaluated a series of complex mixed gambles, each consisting of probabilistic outcomes that spanned monetary losses and gains (Fig. 1A). Volunteers were given an opportunity to improve each gamble by adding money to one of the outcomes in one of three ways: increasing the magnitude of the highest gain $\left(G_{\max }\right)$, decreasing the magnitude of the worst loss $\left(L_{\min }\right)$, or improving the overall probability of winning $\left(P_{\max }\right)$ by adding money to a central reference outcome (Venkatraman et al., 2009) (Fig. $1 B$ ).

To assess changes in risk preference in the absence of learning, participants first made their decisions without feedback. Then, to ascertain changes in response to reward (or loss), a subset of gambles was resolved for real monetary rewards or losses at the end of the experiment (Fig. 1C). Separating the decision and outcome phases could be important as sleep deprivation might interact with task context to influence neural responses and behavior. For example, the propensity to take higher risks in a gambling task was found to be modulated by decision frames (McKenna et al., 2007) and task differences may contribute to lowered (Killgore et al., 2008) or increased (Killgore et al., 2006; Venkatraman et al., 2007) risky decisions.

Finally, we evaluated the extent to which sleep deprivation jointly alters sustained attention along with risky decisionmaking. Although SD may have reproducibly different effects on subjective measures of fatigue, vigilance and other cognitive functions (Van Dongen et al., 2004), decision making has not been evaluated together with other cognitive functions in the same experiment. Existing countermeasures against SD have only been shown to consistently benefit vigilant attention (Wesensten et al., 2005; Killgore et al., 2007). If decision making were to be affected independent of effects on vigilance, prevailing beliefs concerning the generalized utility of existing countermeasures would merit reappraisal. 


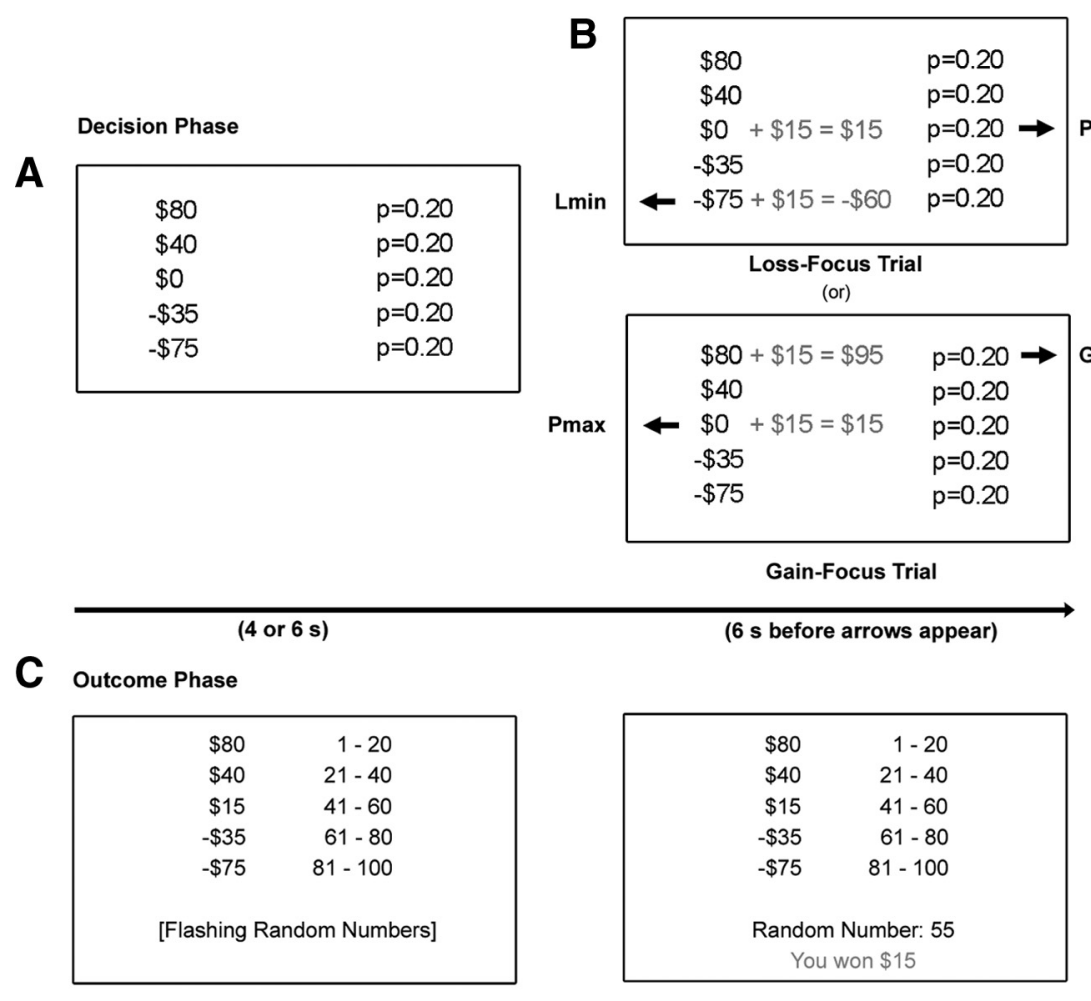

$(2,4$ or $6 \mathrm{~s})$

(2s)

Figure 1. Schematic of the experimental stimuli. A, Participants were first shown a five-outcome mixed gamble, where each outcome was associated with a probability of occurrence. This base gamble was displayed for 4 or $6 \mathrm{~s}$. B, They were then presented with two options for improving the gambles, and had $6 s$ to choose between these. In the gain-focus trials, they chose between adding money to the extreme gain $\left(G_{\max }\right)$ or to the reference outcome, which changed the overall probability of winning $\left(P_{\max }\right)$. In the loss-focus trials, they chose between adding money to the extreme loss (loss-minimizing, $L_{\min }$ ) outcome or to the $P_{\max }$ outcome. After the $6 \mathrm{~s}$ decision phase, two arrows identified the buttons corresponding to each choice and participants indicated their preference by pressing the appropriate button as quickly as possible. A fresh trial appeared after a variable interval of 4,6 or $8 \mathrm{~S}$, with no feedback given after each trial. C, A subset of modified gambles was resolved at the end of the experiment. The five-outcome gamble was displayed with probabilities translated to numbers from 1 to 100 . A random number identifying the winning outcome changed rapidly at the bottom of the screen. After a variable delay of $2-6 \mathrm{~s}$, the number generated identified the winning outcome for that gamble. A message indicating the outcome was displayed for $2 \mathrm{~s}$, followed by a variable interval of 2,4 or $6 \mathrm{~s}$ before the next trial.

\section{Materials and Methods}

Twenty-nine healthy young adults (mean age 22.34 years, standard deviation 1.23 years, 15 males) participated in this neuroimaging study. All participants provided informed consent, and the study was approved by the Singapore General Hospital Institutional Review Board.

\section{Procedure}

Participants visited the lab three times over 2 weeks. On the first visit, participants provided informed consent and practiced the in-scanner task. At the end of this session, participants were instructed to maintain regular sleeping hours throughout the study duration, verified using wrist actigraphy (Philips Respironics). Participants returned to the lab weekly for two functional magnetic resonance imaging sessions: a rested-wakefulness (RW) session and a SD session (order counterbalanced).

Scans at RW took place at 8:00 A.M. For the SD session, participants were monitored in the laboratory from 6:00 P.M. onwards, and scanning took place at 6:00 A.M. the next day. They were allowed to engage in nonstrenuous activities such as reading, watching videos, and conversing.

Before each scan, participants confirmed that they had not smoked or consumed any medications, stimulants, alcohol, or caffeine for at least $24 \mathrm{~h}$ before the scan. For 10 min every hour from 7:00 P.M. until 5:00 A.M., participants completed the Psychomotor Vigilance Task (Dinges et al., 1997; Doran et al., 2001), an extensively used test of sustained attention.
Within each scanning session, participants performed three different tasks. First, they completed six runs of a risky decision-making task, each run comprising 20 gambles and lasting $6 \mathrm{~min}$. Following these decision runs, they completed one run of a Counting Stroop task (data not discussed here). Finally, to evaluate neural sensitivity to rewards, participants watched passively while a subset of modified gambles from the decision runs was resolved to gains and losses during a single outcome run, lasting $7 \mathrm{~min}$.

Stimuli were created using the Psychophysics Toolbox for MATLAB (MathWorks). At the beginning of the experiment, participants were briefed on the incentive-compatible payment procedure and were told that the study did not involve deception. Two outcomes (one from each session, RW and SD) were randomly selected and scaled for bonus payment (see supplemental materials, available at www. jneurosci.org). All participants acknowledged their understanding and acceptance of these procedures.

\section{Experimental tasks and stimuli}

In the risky decision-making task, participants evaluated a series of 120 five-outcome gambles, each of which posed choices that ranged from large monetary losses to large monetary gains (Fig. $1 A$ ). Each gamble comprised two positive outcomes (an extreme outcome of $\$ 65$ to $\$ 80$; an intermediate outcome of $\$ 35$ to $\$ 50$ ), two negative outcomes (an extreme outcome of $-\$ 65$ to $-\$ 85$; an intermediate outcome of $-\$ 35$ to $-\$ 50)$, and a central, reference outcome. Probabilities of each of the five outcomes varied between 0.1 and 0.3 in units of 0.05 , and always summed to 1 across the five outcomes.

Each trial began with the display of a fiveoutcome gamble for 4 or $6 \mathrm{~s}$. Participants were instructed to examine each gamble as it was presented. In the gain-focus trials, participants could make one of two possible choices: a gainmaximizing $\left(G_{\max }\right)$ choice that involved increasing the magnitude of the highest gain, and a probabilitymaximizing $\left(P_{\max }\right)$ choice that improved the overall probability of winning money compared to losing money. Conversely, in the lossfocus trials, participants decided between a loss-minimizing $\left(L_{\min }\right)$ choice that involved decreasing the magnitude of the worst loss, and the $P_{\max }$ choice (Fig. $1 \mathrm{~B}$ ). The relative change in expected value associated with the two choices was varied across trials, both to allow assessment of the consistency of participant preferences and to increase the likelihood that participants would attend to task information (see supplemental materials, available at www.jneurosci.org).

The amount that participants could add to the outcomes ranged between $\$ 10$ and $\$ 25$ and could differ between the two outcomes. All outcome values used in this experiment were multiples of $\$ 5$. Expected values of the two choices were systematically manipulated by changing the amount and/or probabilities associated with each of the options. The amount that could be added and the resulting modified outcome values were displayed in red for both choices, to minimize individual differences that could arise from calculation or estimation biases.

Participants viewed the two options without any response cues for $6 \mathrm{~s}$, whereupon arrows appeared on the screen to indicate which button corresponded to each option. They were instructed to respond as quickly as possible when the arrows appeared. The next trial appeared after a variable intertrial interval of 4,6 , or $8 \mathrm{~s}$. No feedback was given after 
each trial, thus precluding any SD-related changes in trial-to-trial learning from influencing behavior.

In the final run, 40 of the gambles from the decision phase were randomly selected and resolved to an actual monetary gain or loss. These gambles were presented in modified form based on the participants' earlier decisions (Fig. 1C). On each trial, participants passively viewed a gamble on the screen for 2-6s (anticipation phase) while random numbers flashed at the bottom of the screen before stopping at a particular value. The amount won or lost was displayed for $2 \mathrm{~s}$, followed by an intertrial fixation period of 2, 4 or $6 \mathrm{~s}$ before the onset of the next trial.

Participants viewed the stimuli using magnetic resonance-compatible liquid crystal display visual goggles (Resonance Technologies) and responded using a button box held in their right hand.

\section{Imaging procedure}

Magnetic resonance imaging was performed using a 3T Siemens Tim Trio system. For all runs, a single-shot, gradient-echo echoplanar imaging sequence was used (repetition time, $2000 \mathrm{~ms}$; echo time, $30 \mathrm{~ms}$; flip angle, $90^{\circ}$; field of view, $192 \times 192 \mathrm{~mm}$; matrix, $64 \times 64$ ). Parallel imaging (GRAPPA) was enabled. One hundred eighty volumes were collected in each decision run and 220 in the outcome run. Thirty-six oblique axial slices ( $3 \mathrm{~mm}$ thick with $0.3 \mathrm{~mm}$ interslice gap) approximately parallel to the intercommissural plane were acquired. Highresolution coplanar T1 anatomical images were also obtained. For the purpose of image display on Talairach space, 3D high-resolution anatomical reference images were acquired using a T1-weighted magnetizationprepared rapid-acquisition gradient echo sequence.

\section{Data analysis}

Behavioral data. The proportions of $G_{\max }$ or $L_{\min }$ decisions, relative to $P_{\max }$ decisions, were computed for the gain-focus and loss-focus trials respectively. Effects of condition, state and their interaction were investigated using a 2 by 2 repeated-measures ANOVA with factors of choice $\left(G_{\max }, L_{\min }\right)$ and state $(\mathrm{RW}, \mathrm{SD})$.

Imaging data. Analysis was performed using FEAT (FMRI Expert Analysis Tool) Version 5.63, part of the FSL (FMRIB's Software Library, www.fmrib.ox.ac.uk/fsl) package (Smith et al., 2004). The following prestatistics processing steps were applied: motion correction using MCFLIRT, slice-timing correction, removal of non-brain voxels using BET, spatial smoothing with a Gaussian kernel of full-width halfmaximum $8 \mathrm{~mm}$, and high-pass temporal filtering. Registration to high resolution and standard images was performed using FLIRT.

Analysis of the decision phase focused on two regressors for each state that modeled the two types of decision trials: gain-focus and loss-focus. We included additional predictors that modeled the initial presentation of the wager as well as the response period (scaled by reaction time). Data from multiple runs collected for each participant were used to generate subject-specific contrast maps using fixed-effects analysis.

Analysis of the outcome phase involved the use of three regressors in each state to model the anticipation phase (as participants were waiting for the corresponding outcome to be revealed), non-negative outcomes (gain and zero gain), and negative outcomes (loss).

Each of the three regressors was generated by convolving the impulse at the onset of events of interest with a double-gamma function. Higher level across-participants statistical maps were generated using FSL's linear analysis of mixed effects (FLAME stage 1 only) tool, using a cluster threshold of $z>2.3$ and a whole-brain corrected cluster significance threshold of $p<0.05$. We used two-sample paired $t$ tests at the higher level FLAME analysis for estimating state effects. Regions of interest (ROIs) were functionally defined based on the thresholded statistical maps. The parameter estimates from these ROIs were then correlated with the behavioral measures.

\section{Results}

\section{Behavioral findings}

A 2 (choice: $G_{\max }, L_{\min }$ ) by 2 (state: RW, SD) repeated-measures ANOVA showed no main effects of state or choice, but a significant state-by-choice interaction $\left(F_{(1,28)}=8.71, p<0.01\right)$. Sleepdeprived participants exhibited an increased tendency to seek gain, as evidenced by an increased proportion of $G_{\max }$ vs $P_{\max }$ choices in gain-focus trials $\left(t_{(28)}=2.18, p<0.05\right)$, together with a lower proportion of $L_{\min }$ vs $P_{\max }$ choices in loss-focus trials $\left(t_{(28)}=2.05, p<0.05\right.$, supplemental Fig. 1, available at www. jneurosci.org as supplemental material). SD also resulted in increased response times during the response phase of the decisionmaking task $\left(t_{(28)}=5.61, p<0.001\right)$. Importantly, participants remained sensitive to the expected-value relationship between the two choices in both states, indicating that SD led to a change in preferences, not a simple increase in decision variability (supplemental Fig. 2, available at www.jneurosci.org as supplemental material).

$\mathrm{SD}$ also resulted in reduced psychomotor vigilance, a measure of sustained attention, as indexed by increased average response times $\left(t_{(28)}=2.7, p<0.05\right)$ and lapses $\left(t_{(28)}=3.42, p<0.01\right)$ in the Psychomotor Vigilance Task. Critically, this decline in sustained attention did not correlate with changes in economic preference.

We next estimated a risk parameter $(\alpha)$ and loss-aversion parameter $(\lambda)$ for each subject in each state (see supplemental materials, available at www.jneurosci.org). Participants were more risk-seeking following $\mathrm{SD}\left(\alpha_{\mathrm{RW}}=0.95, \alpha_{\mathrm{SD}}=1.10, t_{(28)}=2.16\right.$, $p<0.05)$, consistent with an increased tendency to chase large gains. Importantly, this parameter was positively correlated with proportion of $G_{\max }$ choices $\left(r_{\mathrm{RW}}=0.6, r_{\mathrm{SD}}=0.46\right)$ and negatively correlated with proportion of $L_{\min }$ choices in both states $\left(r_{\mathrm{RW}}=-0.62, r_{\mathrm{SD}}=-0.58\right)$. There was no significant difference in the loss-aversion parameters across state. Thus, the effects of $\mathrm{SD}$ on multi-outcome decisions can be attributed to changes in decision parameters or an overall shift in choice bias (without recourse to any specific model). Previous studies using a similar multi-outcome risky choice task suggest that using standard decision parameters to predict behavior leads to specific errors; e.g., not accounting for the high proportion of $P_{\max }$ choices (Payne, 2005; Venkatraman et al., 2009). Thus, we hereafter use proportions of choices as covariates in subsequent analyses, noting that all resulting effects may also be consistent with an overall increase in a risk-seeking parameter.

\section{Neuroimaging findings}

\section{Decision phase}

Across both states, consistent with prior studies of risky decision making (Huettel et al., 2006; Platt and Huettel, 2008; Rangel et al., 2008), we observed task-related increases in bilateral intraparietal sulcus, dorsolateral prefrontal cortex (dlPFC), anterior insula (aINS) and dorsomedial prefrontal cortex (dmPFC) activation (supplemental Table 1, available at www.jneurosci.org as supplemental material).

After a night of normal sleep, higher activity in the right aINS for loss-focus trials correlated with increased preference for the $L_{\min }$ option $(r=0.43, p<0.05)$, while increased activity (in this case, reduced deactivation) in the ventromedial prefrontal cortex (vmPFC) for gain-focus trials correlated with an increased proportion of $G_{\max }$ choices ( $r=0.43, p<0.05$, supplemental Fig. 3, available at www.jneurosci.org as supplemental material). These findings concur with previous studies associating aINS activation with loss-averse behavior (Paulus et al., 2003; Kuhnen and Knutson, 2005; Venkatraman et al., 2009) and vmPFC activation with gain-seeking behavior (Bechara et al., 2000; Tobler et al., 2007; Venkatraman et al., 2009). Additionally, we observed that activation in the vmPFC for gain-focus trials following normal sleep correlated positively with the risk parameter $\alpha_{\mathrm{RW}}(r=0.45$, $p<0.001)$ suggesting that our choice-based interpretations 


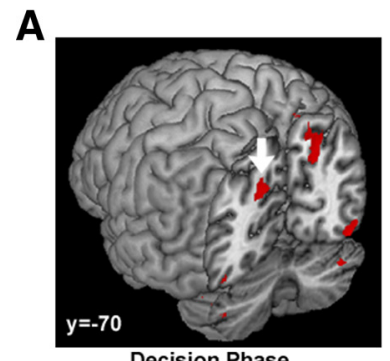

Decision Phase

RW > SD
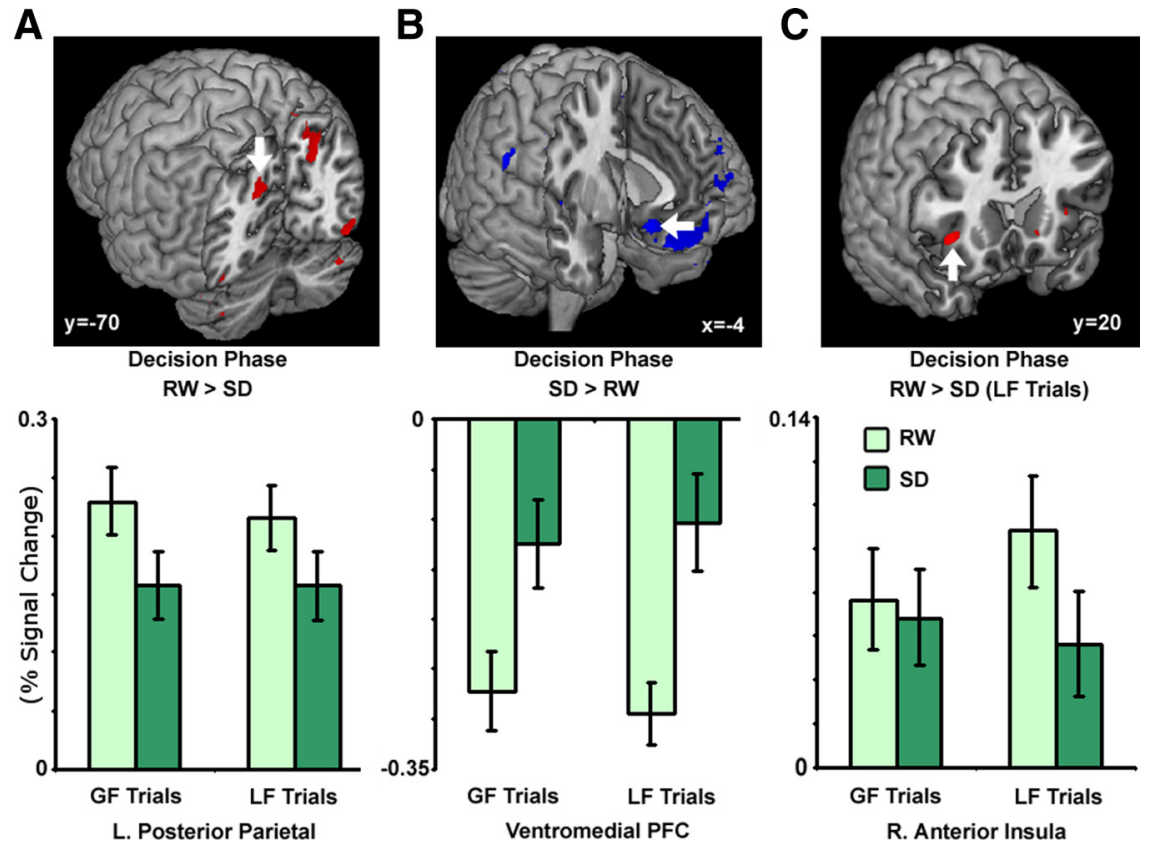

SD $>$ RW

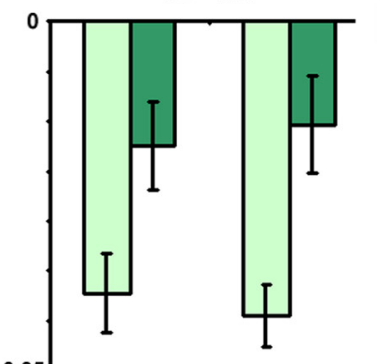

GF Trials LF Trials
Ventromedial PFC

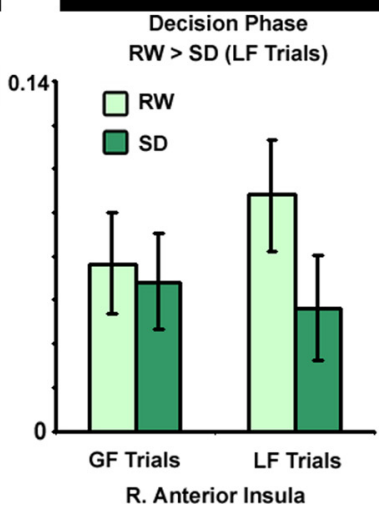

Figure 2. Sleep deprivation affects neural systems underlying decision making. $\boldsymbol{A}$, Across all trials, SD led to reduced activation in bilateral intraparietal sulcus [peak MNI (Montreal Neurological Institute) coordinates: $x=20, y=-74, z=50$; indicated with arrow] and right thalamus ( $x=14, y=-8, z=-3$; data not shown in the figure). $\boldsymbol{B}$, SD resulted in increased activation (in this case, reduced deactivation) in the ventromedial prefrontal cortex ( $x=-1, y=18, z=-14$; indicated by an arrow). C, SD was also associated with reduced activation in right anterior insula ( $x=39, y=19, z=2$; indicated by an arrow) and dorsomedial prefrontal cortex $(x=1, y=33, z=48$; data not shown in the figure) for the loss-focus trials. Activation maps show active clusters that surpassed a threshold of $z>2.3$ using cluster-based Gaussian random field correction. On this and subsequent figures, error bars in the percentage signal change plots represent \pm 1 SEM.
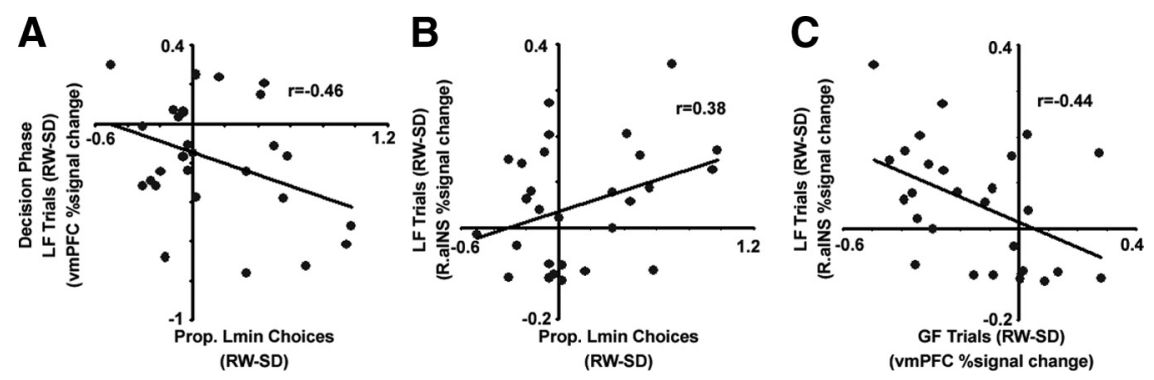

Figure 3. Changes in right (R.) alNS and vmPFC activation predict shifts in preferences following sleep deprivation. $A$, Following $S D$, decreased activation in the right anterior insula correlated with a decrease in proportion of $L_{\min }$ choices. $\boldsymbol{B}$, Increased activation in vmPFC following SD during loss-focus trials correlated negatively with decreased preference for $L_{\min }$ choices. $C$, The decrease in activation in the right anterior insula during loss-focus trials correlated significantly with increased vmPFC activation during gain-focus trials.

would be largely consistent even when using a parametric approach.

Sleep deprivation resulted in reduced activation of bilateral intraparietal sulci, and increased activation in vmPFC (Fig. $2 A, B$ ) and right thalamus (supplemental Table 2, available at www.jneurosci.org as supplemental material). SD-related reduction of task-related parietal cortex activation has been extensively reported (Bell-McGinty et al., 2004; Chee and Chuah, 2007; Chee et al., 2008; Tomasi et al., 2009) and is not discussed further in this article.

In addition to the main effects described above, sleep deprivation led to reduced activation in the right anterior insula (Fig. 2C) and dmPFC during loss-focus trials (supplemental Table 2, available at www.jneurosci.org as supplemental material). Notably, these SD-induced changes in activation correlated with SD-induced changes in behavior. A reduced propensity to make $L_{\text {min }}$ choices when sleep deprived correlated with reduced right anterior insula activation during these trials $(r=$ $0.38, p<0.05$, Fig. $3 A$ ). There was also a significant negative correlation between state-related changes in the proportion of $L_{\text {min }}$ choices and changes in vmPFC activation $(r=0.46, p<0.01$, Fig. $3 B)$, driven possibly by the increased focus on the higher ranked $P_{\max }$ choices during these trials following SD. The SD-related decrease in activation in the right anterior insula for loss-focus trials correlated positively, across participants, with an increase in activation in the vmPFC for the gain-focus trials $(r=$ $-0.44, p<0.01$, Fig. 3C).

SD did not affect dlPFC activation, even at an uncorrected threshold of $p<$ 0.005 .

\section{Outcome phase}

After making a series of decisions about risky gambles, each participant passively viewed a subset of those gambles being resolved to an actual monetary gain or loss. Activity in the ventral striatum (vStr) and vmPFC increased for gains relative to losses across both sessions, consistent with the association of these regions with reward processing (Breiter et al., 2001; Knutson et al., 2003; Seymour et al., 2007). No region showed significantly greater activation for losses over gains in either session.

Following SD, there was a significant increase in gain-related activity in the vStr and vmPFC compared to after a normal night of sleep (Fig. 4). Conversely, SD was associated with marked attenuation of loss-related activation within the left anterior insula (Fig. 5). Changes in anterior insula activation occurred at the identical location to where sleep-deprived persons showed attenuated responses to losses in an earlier study (Venkatraman et al., 2007). Finally, the decrease in activation of the left anterior insula for losses correlated with the increase in activation in ventral striatum for gains $(r=-0.45, p<$ 0.05 , supplemental Fig. 4, available at www.jneurosci.org as supplemental material).

Notably, there were no significant correlations between stateinduced changes in the decision and outcome phases in any region of interest, suggesting that SD affects the processes underlying these two phases differently across individuals.

\section{Discussion}

Using a risky decision-making task, we showed that sleep deprivation shifted most persons' bias from avoiding loss to pursuing gain. This behavioral change accompanied congruent alterations of activation in brain regions associated with reward anticipation and emotional processing. Additionally, we observed altered neural sensitivity to received gains and losses that were directionally similar to but uncorrelated with altered activation associated 
with decision making. Finally, the magnitude of SD-induced shifts in risky decision-making strategies was not correlated with SD-induced reductions in psychomotor vigilance.

\section{Sleep deprivation favors the pursuit of gain}

The current study is the first to link SDinduced changes in economic behavior with changes in brain activation. A previous study found that SD altered activation in reward-related regions in the absence of any significant differences in choice preferences (Venkatraman et al., 2007). Since that study only contrasted gambles with positive outcomes to mixed gambles involving both positive and negative out-

comes, the SD-related alteration of risk preferences could have been masked by the tendency to maximize the overall probability of winning (Payne, 2005; Venkatraman et al., 2009). The present study exclusively used mixed gambles and separated decision and outcome phases, facilitating the identification of state-related alterations in economic preferences.

While well rested participants sought to minimize the effect of the worst loss, SD caused the same individuals to be less concerned about losses and to shift to a strategy that improved the magnitude of the best gain. In the absence of explicit feedback, this behavioral change suggests an unfounded rise in expectation for gain as reflected in a systematic bias in economic preferences following sleep deprivation.

Sleep deprivation altered vmPFC responses in both decision and outcome phases of the present study. The vmPFC has been shown to be instrumental in computing value as well as in supporting processes related to learning from reward and punishment (Rolls et al., 1994; Bechara et al., 2000; Rangel et al., 2008). One explanation for the effects of SD on vmPFC activation is that SD could hamper the integration of feedback when making decisions, consistent with the role of this region in learning. However, this seems unlikely, given that there was no explicit feedback at the end of each trial, and given the relative preservation of dlPFC engagement during SD.

A more likely alternative is that SD influences valuation. Though several studies associate vmPFC activation with the implicit overall value of the gamble (Tom et al., 2007; Levy et al., 2010), participants in this task may frequently be making decisions without computing an integrative value signal that incorporates all aspects of the gamble. Instead, there may be systematic biases in the attention paid to different aspects of the gamble like probabilities, highest gain and worst loss. In this context, we argue that elevated vmPFC activation is predictive of biases placed on the higher-ranked outcomes (Venkatraman et al., 2009). Our findings here fit well with this latter viewpoint, since the SDrelated change in vmPFC activation during decision making also correlated with an increased focus on the highest gains. Further, during loss-focus trials, an increase in vmPFC activation following SD correlated with a decreased preference for the $L_{\text {min }}$ choices, suggesting a reduced focus on the lower-ranked $L_{\text {min }}$ options in favor of higher-ranked $P_{\max }$ choices within these trials. Together, these findings suggest that SD-induced increases in vmPFC activation might underlie the bias toward selecting higher-ranked outcomes when one is sleep deprived.
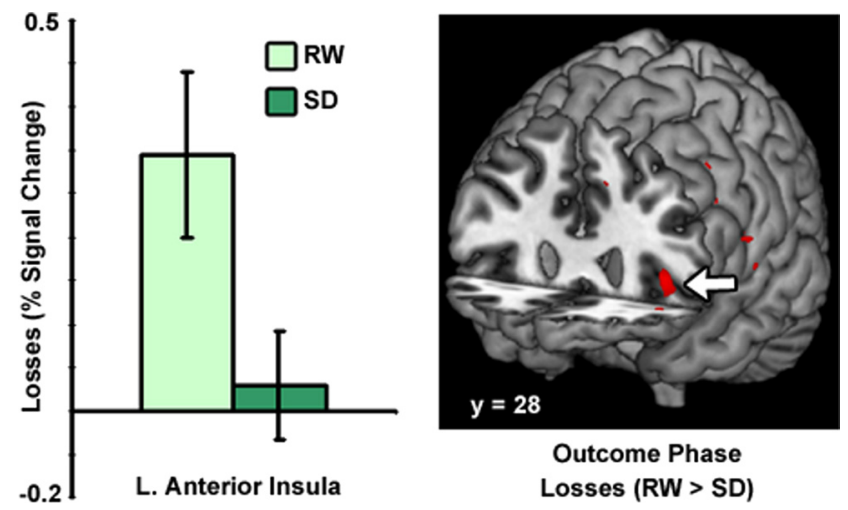

Figure 5. Sleep deprivation diminishes neural sensitivity to loss outcomes. Following sleep deprivation, there was marked attenuation of loss-related activation within the left anterior insula ( $x=-40, y=30, z=1$; indicated by the arrow).

\section{Sleep deprivation reduces the minimization of loss}

$\mathrm{SD}$ led to decreased activation in the right anterior insula and $\mathrm{dmPFC}$ during decisions that involved loss-focus trials. Activation in these regions has been widely observed in studies involving emotional awareness, particularly those relating to negative affect (Dalgleish, 2004; Craig, 2009; van Veen et al., 2009). Consistent with these observations, we found that activation in anterior insula was positively correlated with $L_{\min }$ choices in well rested participants. SD lowered right anterior insula activation, and this correlated with a decrease in preference for the $L_{\text {min }}$ choices.

A natural alternative hypothesis is that SD introduces noise into the decision process, given that under SD participants were equally likely to make $L_{\min }$ or $P_{\max }$ choices on loss-focus trials. However, several aspects of the data make such an explanation unlikely: (1) Under SD, the proportion of $G_{\max }$ choices increased and was significantly greater than chance $\left(\chi^{2}=16, p<0.001\right)$, (2) participants showed significant variability in choice preferences; yet the proportion of $L_{\text {min }}$ choices varied systematically between states across participants $(r=0.36, p<0.06$, supplemental Fig. 5, available at www.jneurosci.org as supplemental material), (3) preferences following sleep deprivation remained sensitive to the expected-value relationship between the two choices, and (4) activation levels in dorsolateral prefrontal cortex (associated with cognitive control and executive function) did not vary as a function of sleep deprivation. Thus, changes in preferences provide a more parsimonious explanation for the observed results. 
Considered together, SD appears to create an optimism bias; for example, participants behave as if positive consequences are more likely (or more valuable) and as if negative consequences are less likely (or less harmful). In support of this general interpretation, we found that the SD-related decrease in anterior insula activation associated with loss-focus trials correlated with SD-related increases in vmPFC activation during gain-focus trials. As activation in these regions are typically associated with salience of negative and positive outcomes respectively (Kuhnen and Knutson, 2005; Preuschoff et al., 2008; Venkatraman et al., 2009), it appears that SD biases valuation by bringing about an increased attentional bias toward higher-ranked positive outcomes while concurrently reducing concern for losses.

\section{Effects of SD: choices or parameters?}

In this study, we identified brain systems whose activation correlated with different choices or with choice tendencies. An alternative yet popular approach in decision neuroscience involves estimating parameters using decision theoretic models like prospect theory and identifying brain functions that track changes in that parameter across individuals. However, in several studies using a similar multi-outcome risky choice task (but without a state manipulation), participants' showed a strong bias toward $P_{\max }$ choices, which is inconsistent with traditional economic models like expected utility theory and prospect theory. Instead, individuals appear to adopt an aspiration level that emphasizes selected aspects of the decision problems that in turn bias choices (Payne, 2005; Venkatraman et al., 2009, 2011).

Since sleep deprivation did not lead to changes in the proportion of $P_{\max }$ choices, the results in the current experiment are agnostic to model-based and model-free methods. Not surprisingly, analyzing choices using a parametric approach indicates that $\mathrm{SD}$ still changes the risk but not the loss-aversion parameter. Moreover, SD-induced changes in choices were highly correlated with SD-induced changes in the risk parameter across participants. Thus, SD alters underlying decision preferences, whether expressed in increased tendency for gain-seeking choices or in a change in parameter of models of risk. One conjecture is that SD modulates these preferences by changing the relative emphasis paid to different aspects of the complex problem presented; specifically, by increasing the biases toward gains relative to losses. This would be consistent with prior work showing that framing a risky decision as a gain or as a loss leads to different patterns of choice following SD (McKenna et al., 2007).

\section{SD independently amplifies optimism bias during the outcome phase}

When a subset of gambles was resolved to gains or losses at the end of the experiment, sleep-deprived participants showed increased activation of ventral striatum and vmPFC following gain outcomes and decreased activation in anterior insula when gambles were resolved to losses. The attenuated anterior insula activation for losses mirrors previous findings concerning neural responses at the outcome phase (Venkatraman et al., 2007). Notably, in the present experiment, the effects of SD on decision and outcome phases of the task were not correlated.

In a real-world parallel, sleep-deprived gamblers and traders, already saddled with an optimism bias during decision making, could further compound gain-seeking, high-risk behavior by being disproportionately incentivized toward reward-seeking while concurrently being desensitized to ongoing losses. More positively, because SD has dissociable effects on decision making and response to outcomes, some sleep-deprived persons may tran- scend the state-induced optimism bias and respond more appropriately to losses as they occur.

\section{Potential mechanisms underlying SD-related optimism bias}

Risky choice often involves weighting potential gains against potential losses. Our findings suggest that SD might bias value computations by increasing the emphasis on the gain outcomes, relative to losses (Kuhnen and Knutson, 2005; Liu et al., 2007). Strikingly, the shifts in economic preferences were independent of the effects of SD on vigilance, suggesting that SD's influence on behavior could vary according to cognitive domain (Van Dongen et al., 2004). This is particularly relevant in light of increasing number of persons seeking to maintain performance when sleepdeprived by taking stimulants. Stimulants may improve vigilance but their influence on other aspects of cognition, such as decision making, is less clear (Gottselig et al., 2006; Killgore et al., 2007, 2008; Huck et al., 2008). Our findings that SD shapes decision preferences independent of its effects on vigilance suggest that the traditional countermeasures may be ineffective in ameliorating the decision biases engendered by limited sleep.

Future experiments should consider cross-sectional heterogeneity in the effects of SD on preferences as interindividual variation in the effect of sleep deprivation on attention has been shown to be trait like (Van Dongen et al., 2004). The behavior-imaging correlates of such variation have been shown in several imaging studies (Mu et al., 2005; Lim et al., 2007; Chee and Chuah, 2008; Chee and Tan, 2010). Here, on top of significant main effects of $\mathrm{SD}$, closer inspection revealed substantial variability in its effects on individuals' choices and brain activation (Fig. 3; supplemental Fig. 6, available at www.jneurosci.org as supplemental material). While there was an overall increase in preference for $G_{\max }$ choices following $\mathrm{SD}$, there was a subset of participants who were biased in the other direction.

We conjecture that changes in dopamine neurotransmission following SD may affect decision making in that state. In a recent study, healthy sleep-deprived persons showed elevations in dopamine levels in the striatum and thalamus, thought to contribute to maintaining wakefulness, albeit in a somewhat maladaptive way given the concurrent decline in visual attention as volunteers engaged in task performance (Volkow et al., 2008, 2009). As L-Dopa administered to healthy young adults can transiently elevate subjective ratings of pleasantness (Sharot et al., 2009), we speculate that the optimism bias observed in SD could be a byproduct of attempts to sustain wakefulness by elevating dopamine levels. The varying extent to which this is successful in a given individual might then account for the dissociation between vigilance and shift in risk preference.

\section{References}

Bechara A, Damasio H, Damasio AR (2000) Emotion, decision making and the orbitofrontal cortex. Cereb Cortex 10:295-307.

Bell-McGinty S, Habeck C, Hilton HJ, Rakitin B, Scarmeas N, Zarahn E, Flynn J, DeLaPaz R, Basner R, Stern Y (2004) Identification and differential vulnerability of a neural network in sleep deprivation. Cereb Cortex 14:496-502.

Breiter HC, Aharon I, Kahneman D, Dale A, Shizgal P (2001) Functional imaging of neural responses to expectancy and experience of monetary gains and losses. Neuron 30:619-639.

Centers for Disease Control and Prevention (2009) Perceived insufficient rest or sleep among adults - United States, 2008. MMWR Morb Mortal Wkly Rep 58:1175-1179.

Chee MW, Choo WC (2004) Functional imaging of working memory after $24 \mathrm{hr}$ of total sleep deprivation. J Neurosci 24:4560-4567.

Chee MW, Chuah YM (2007) Functional neuroimaging and behavioral cor- 
relates of capacity decline in visual short-term memory after sleep deprivation. Proc Natl Acad Sci U S A 104:9487-9492.

Chee MW, Chuah LY (2008) Functional Neuroimaging insights into how sleep and sleep deprivation affect memory and cognition. Curr Opin Neurol 21:417-423.

Chee MW, Tan JC (2010) Lapsing when sleep deprived: neural activation characteristics of resistant and vulnerable individuals. Neuroimage 51:835-843.

Chee MW, Tan JC, Zheng H, Parimal S, Weissman DH, Zagorodnov V, Dinges DF (2008) Lapsing during sleep deprivation is associated with distributed changes in brain activation. J Neurosci 28 5519-5528.

Craig AD (2009) How do you feel-now? The anterior insula and human awareness. Nat Rev Neurosci 10:59-70.

Dalgleish T (2004) The emotional brain. Nat Rev Neurosci 5:583-589.

Dinges DF, Pack F, Williams K, Gillen KA, Powell JW, Ott GE, Aptowicz C, Pack AI (1997) Cumulative sleepiness, mood disturbance, and psychomotor vigilance performance decrements during a week of sleep restricted to 4-5 hours per night. Sleep 20:267-277.

Doran SM, Van Dongen HP, Dinges DF (2001) Sustained attention performance during sleep deprivation: evidence of state instability. Arch Ital Biol 139:253-267.

Drummond SP, Meloy MJ, Yanagi MA, Orff HJ, Brown GG (2005) Compensatory recruitment after sleep deprivation and the relationship with performance. Psychiatry Res 140:211-223.

Gottselig JM, Adam M, Rétey JV, Khatami R, Achermann P, Landolt HP (2006) Random number generation during sleep deprivation: effects of caffeine on response maintenance and stereotypy. J Sleep Res 15:31-40.

Habeck C, Rakitin BC, Moeller J, Scarmeas N, Zarahn E, Brown T, Stern Y (2004) An event-related fMRI study of the neurobehavioral impact of sleep deprivation on performance of a delayed-match-to-sample task. Brain Res Cogn Brain Res 18:306-321.

Harrison Y, Horne JA (1999) One night of sleep loss impairs innovative thinking and flexible decision making. Organ Behav Hum Decis Process 78:128-145.

Harrison Y, Horne JA (2000) The impact of sleep deprivation on decision making: a review. J Exp Psychol Appl 6:236-249.

Huck NO, McBride SA, Kendall AP, Grugle NL, Killgore WD (2008) The effects of modafinil, caffeine, and dextroamphetamine on judgments of simple versus complex emotional expressions following sleep deprivation. Int J Neurosci 118:487-502.

Huettel SA, Stowe CJ, Gordon EM, Warner BT, Platt ML (2006) Neural signatures of economic preferences for risk and ambiguity. Neuron 49:765-775.

Institute of Medicine (2006) Sleep disorders and sleep deprivation: an unmet public health problem. Washington DC: The National Academies Press.

Killgore WD, Balkin TJ, Wesensten NJ (2006) Impaired decision making following $49 \mathrm{~h}$ of sleep deprivation. J Sleep Res 15:7-13.

Killgore WD, Lipizzi EL, Kamimori GH, Balkin TJ (2007) Caffeine effects on risky decision making after 75 hours of sleep deprivation. Aviat Space Environ Med 78:957-962.

Killgore WD, Grugle NL, Killgore DB, Leavitt BP, Watlington GI, McNair S, Balkin TJ (2008) Restoration of risk-propensity during sleep deprivation: caffeine, dextroamphetamine, and modafinil. Aviat Space Environ Med 79:867-874.

Knutson B, Fong GW, Bennett SM, Adams CM, Hommer D (2003) A region of mesial prefrontal cortex tracks monetarily rewarding outcomes: characterization with rapid event-related fMRI. Neuroimage 18:263-272.

Kuhnen CM, Knutson B (2005) The neural basis of financial risk taking. Neuron 47:763-770.

Levy I, Snell J, Nelson AJ, Rustichini A, Glimcher PW (2010) Neural representation of subjective value under risk and ambiguity. J Neurophysiol 103:1036-1047.

Lim J, Choo WC, Chee MW (2007) Reproducibility of changes in behaviour and fMRI activation associated with sleep deprivation in a working memory task. Sleep 30:61-70.

Linde L, Edland A, Bergstrom M (1999) Auditory attention and multiattribute decision-making during a $33 \mathrm{~h}$ sleep-deprivation period: mean performance and between-subject dispersions. Ergonomics 33:696-713.

Liu X, Powell DK, Wang H, Gold BT, Corbly CR, Joseph JE (2007) Func- tional dissociation in frontal and striatal areas for processing of positive and negative reward information. J Neurosci 27:4587-4597.

McKenna BS, Dickinson DL, Orff HJ, Drummond SP (2007) The effects of one night of sleep deprivation on known-risk and ambiguous-risk decisions. J Sleep Res 16:245-252.

Mu Q, Mishory A, Johnson KA, Nahas Z, Kozel FA, Yamanaka K, Bohning DE, George MS (2005) Decreased brain activation during a working memory task at rested baseline is associated with vulnerability to sleep deprivation. Sleep 28:433-446.

Paulus MP, Rogalsky C, Simmons A, Feinstein JS, Stein MB (2003) Increased activation in the right insula during risk-taking decision making is related to harm avoidance and neuroticism. Neuroimage 19:1439-1448.

Payne JW (2005) It is whether you win or lose: the importance of the overall probabilities of winning or losing in risky choice. J Risk Uncertainty 30:5-19.

Platt ML, Huettel SA (2008) Risky business: the neuroeconomics of decision making under uncertainty. Nat Neurosci 11:398-403.

Preuschoff K, Quartz SR, Bossaerts P (2008) Human insula activation reflects risk prediction errors as well as risk. J Neurosci 28:2745-2752.

Rangel A, Camerer C, Montague PR (2008) A framework for studying the neurobiology of value-based decision making. Nat Rev Neurosci 9:545-556.

Rolls ET, Hornak J, Wade D, McGrath J (1994) Emotion-related learning in patients with social and emotional changes associated with frontal lobe damage. J Neurol Neurosurg Psychiatry 57:1518-1524.

Seymour B, Daw N, Dayan P, Singer T, Dolan R (2007) Differential encoding of losses and gains in the human striatum. J Neurosci 27:4826-4831.

Sharot T, Shiner T, Brown AC, Fan J, Dolan RJ (2009) Dopamine enhances expectation of pleasure in humans. Curr Biol 19:2077-2080.

Smith SM, Jenkinson M, Woolrich MW, Beckmann CF, Behrens TE, Johansen-Berg H, Bannister PR, De Luca M, Drobnjak I, Flitney DE, Niazy RK, Saunders J, Vickers J, Zhang Y, De Stefano N, Brady JM, Matthews PM (2004) Advances in functional and structural MR image analysis and implementation as FSL. Neuroimage 23:S208-S219.

Sterpenich V, Albouy G, Darsaud A, Schmidt C, Vandewalle G, Dang Vu TT, Desseilles M, Phillips C, Degueldre C, Balteau E, Collette F, Luxen A, Maquet P (2009) Sleep promotes the neural reorganization of remote emotional memory. J Neurosci 29:5143-5152.

Tobler PN, O’Doherty JP, Dolan RJ, Schultz W (2007) Reward value coding distinct from risk attitude-related uncertainty coding in human reward systems. J Neurophysiol 97:1621-1632.

Tom SM, Fox CR, Trepel C, Poldrack RA (2007) The neural basis of loss aversion in decision-making under risk. Science 315:515-518.

Tomasi D, Wang RL, Telang F, Boronikolas V, Jayne MC, Wang GJ, Fowler JS, Volkow ND (2009) Impairment of attentional networks after 1 night of sleep deprivation. Cereb Cortex 19:233-240.

Van Dongen HP, Baynard MD, Maislin G, Dinges DF (2004) Systematic interindividual differences in neurobehavioral impairment from sleep loss: evidence of trait-like differential vulnerability. Sleep 27:423-433.

van Veen V, Krug MK, Schooler JW, Carter CS (2009) Neural activity predicts attitude change in cognitive dissonance. Nat Neurosci 12:1469-1474.

Venkatraman V, Chuah YM, Huettel SA, Chee MW (2007) Sleep deprivation elevates expectation of gains and attenuates response to losses following risky decisions. Sleep 30:603-609.

Venkatraman V, Payne JW, Bettman JR, Luce MF, Huettel SA (2009) Separate neural mechanisms underlie choices and strategic preferences in risky decision making. Neuron 62:593-602.

Venkatraman V, Payne JW, Huettel SA (2011) Neuroeconomics of risky decisions: from variables to strategies. In: Decision making, affect, and learning: attention and performance XXIII (Delgado MR, Phelps EA, Robbins TW, eds). Oxford: Oxford UP, in press.

Volkow ND, Wang GJ, Telang F, Fowler JS, Logan J, Wong C, Ma J, Pradhan K, Tomasi D, Thanos PK, Ferré S, Jayne M (2008) Sleep deprivation decreases binding of $\left[{ }^{11} \mathrm{C}\right]$ raclopride to dopamine $\mathrm{D} 2 / \mathrm{D} 3$ receptors in the human brain. J Neurosci 28:8454-8461.

Volkow ND, Tomasi D, Wang GJ, Telang F, Fowler JS, Wang RL, Logan J, Wong C, Jayne M, Swanson JM (2009) Hyperstimulation of striatal D2 receptors with sleep deprivation: implications for cognitive impairment. Neuroimage 45:1232-1240.

Wesensten NJ, Killgore WD, Balkin TJ (2005) Performance and alertness effects of caffeine, dextroamphetamine, and modafinil during sleep deprivation. J Sleep Res 14:255-266. 